\title{
Systematic Literature Survey on Emergency Medical Services
}

\author{
Sharmila Gaikwad ${ }^{1}$, Imran Arkate $^{2}$, Akhumukhi Vidyasagar ${ }^{3}$, Vishal Kumar ${ }^{4}$ \\ Department of Computer Engineering, MCT's Rajiv Gandhi Institute ofTechnology, Mumbai, India. \\ sharmila.gaikwad@mctrgit.ac.in,arkateimran11@gmail.com,sagar112113@yahoo.com,vishal.22@gmail.com
}

\section{Abstract:}

Now-a-days considering the fast-paced world, particularly in massive cities, several services get delayed due to traffic jams. Ambulance service is one among the crucial services that get delayed fairly often. Additionally, generally onsite doctors aren't obtainable. Therefore, the patient doesn't get medical attention forthwith. 'Traffic Control System for Smart Ambulance' is a system which creates a 'green corridor' for the ambulance to reach the hospital. Also, it monitors the patient's health and sends the data to the hospital beforehand. In health monitoring system, the patient's very importanthealth parameters like heart rate and body temperature are monitored. These parameters are sent to the hospital via message. In traffic control an android application is used in which the traffic control room controls the signals based on the traffic conditions and the location of ambulance and hospital seen on the app. The objective of this paper is tounderstand the various systems that can be used for ambulances in traffic and help the ambulance to faster reach the hospital.

Keywords: Green Corridor, Health Monitoring, Smart Ambulance, Traffic Control.

\section{INTRODUCTION}

Population in developing countries such as India is increasing significantly. This result in a number of problems such as heavy traffic jams, violation of the traffic rules and sometimes even accidents. For example, the number of road accidents in major cities such as Mumbai, Chennai, Hyderabad and Delhi increased to 16 deaths per hour, as stated by the Indian Government. The traffic congestion often blocks the path of the emergency vehicles. It is very necessary that emergency vehicles arrive on time to prevent serious loss of human life. A very rapid population growth in cities has resulted in tremendous road traffic within the city. In addition, in recent times the number of deaths due to delays in the arrival of emergency vehicle has risen to greater extent. Hence emergency services such as ambulances must be on time to avoid loss of human life. In the current traffic situation, helping an emergency vehicle move out of traffic congestion is very much important. Now days road accidents are generally increasing due to high population and do not obeying the traffic rules in our country. It is reported that in India more than 150,000 people are died each year in road traffic accidents. So, due to these accidents people are dying but we need to consider other factors like delay in the response of the emergency vehicle like ambulance at the time of accident took place and also delay in the treatment provided to the victims of the accidents in the hospital since the hospital will start to prepare for treatment only after analysing the health condition of the victim . So, these factors also play a vital role in rescuing the life of the victim of the accident. Overall, time is wasted here which is a critical issue that leads to the patient loss. In the current situation of covid 19, the covid cases in india are increasing rapidly, and after the end of lockdown the traffic congestion on roads is at pre covid level, it becomes very important to provide short, traffic free routes to ambulances so that human lives can be saved. 


\section{LITERATURE SURVEY}

Smart Ambulance System by Satyasheel Pol, Dharmanath Rahatekar, Poonam Gupta and Avanti Patil [7] purposed this system in two modules, both modules work on the principle of Iot. Module one is used for finding ambulances and hospital whereas module two is usedfor sending patients information to the hospital. To implement the project the authors have divided system into server client architecture, on launching the app first device will automatically detect the location of user using the GPS devices. Then according to user's requirement, they can choose hospital and ambulances. Then this query is sent in the JSON format to the server. Server will process on it and respond accordingly. The response fromserver will also be in JSON format. Smartphone app will read the data from response and plot the coordinates or information according to the user's request. Server end is designed using php. When server will receive a request from device, it will parse the data and extract result from the database. This result will be placed in the JSON format and will be returned to the device which made that same request. Every JSON request will send data dependingon request URL type.

Internet Controlled Vehicle by Akshay Vishwanath , Lalit Bhosle, Rishab Bhandari and Sharmila Gaikwad [8], purposed a system by using the internet and providing a high end system .It aims at solving problems at various levels in big corporate and other sectors. The taxi service is designed to be with the driver's seat left empty in the vehicle and rather controlled by the driver from a far off distance with the help of a screen $\&$ an emulator connected to the car via Wireless networks. A constant video feed helps the controller obtain real-time footage of the current surroundings of the vehicle. The video feed obtained from the car is then used by the driver to decide which direction the car must now move in. The car is controlled by sending appropriate control signals to it through the internet by means of a web server. The logic controls are provided to the car by the Raspberry Pi processor.

Intelligent Ambulance System by Anupama D.K, Naveen Kulkarni, Shivaleela M.S. [9] purposed this system which is divided into three parts. First part is automatic traffic control. This is done using IR transmitter and receiver, the IR transmitter keeps transmitting IR waves at $38 \mathrm{khz}$. The ambulance which has IR receiver gets the signal. Then wireless communication takes place between the transmitter and receiver and using microcontroller the signal is turned green. In second part the health data of patient is collected such as heart rate, body temperature and is stored in patient database. The third part is biotelemetry, in this part the health data of the patient and database is been telemetered to the hospital through GSM where the doctor receives the message prior to the patient arrival.

Traffic Control System for Smart Ambulance by Bhakti Prabhu, Ruta Deshpande, Shraddha Bhavsar, Shailaja Jha [18] purposed this system which is combination of two systems traffic control and health monitoring. Patient's health parameters are monitored and at the same time driver of the ambulance can request the control room to manipulate the traffic signals. The ambulance will first send a request to the control room for traffic control. The request consists of the current location of the ambulance accessed using GPS and the location of the desired hospital is sent by the ambulance driver. This request is now sent to the AWS server. The server will send the data of the ambulance to the control center. The control room side of the application now displays the requests of the ambulance. Now a map is displayed which shows the current location of the ambulance as well as the location of the hospital. The route between the ambulance and the hospital and the live data of traffic is displayed on the map. Here, green represents less traffic, orange represents moderate traffic and red indicates high traffic. An option is also displayed which enables navigation on google maps. The shortest route between the ambulance and hospital is displayed as well as the traffic at various junctions. With the help of this data, the control room can control the traffic signals at various junctions.

Mobile Agents in Heterogeneous Database Environment for Emergency Healthcare System by Sharmila Gaikwad [13], purposed a system using Multi-database which is one prevalent solution that integrates distributed heterogeneous databases and provides uniform global access methods to the users while preserving local database autonomy. The agent platform is used which can be distributed across machines which not even need to share the same Database or OS and the configuration can be controlled via a remote GUI. The configuration can be 
even changed at run-time by moving agents from one machine to another one, as and when required, the paramedics then utilize the travel time to the scene of emergency to launch a set of two mobile agents. The first mobile agent which is hospital assistant starts off by contacting to discover the hospital nodes that paramedics have short-listed. When these nodes have been successfully discovered, the agent migrates to the firsthospital on its list. Upon arrival, it interacts with the stationary agent present at this node and retrieves the latest resource utilization details for the hospital.

Smart Ambulance Rescue System with Patient Monitoring by Vidya Bangar, Nikita Chaskar, Sayali Kurhade [16] purposed a system in which when the patient clicks on panic button the nearest ambulance available is find out. The emergency request is sent to the driver. When driver accept this request then system define the exact route by using area partitioning and edge upgrading algorithm. Ambulance driver select appropriate hospital based on patient condition. The driver will then send the request to hospital reception staff. Hospital reception assign specific doctor to that patient. Pathology ward send all report of patient to doctor like as blood report, ECG etc.

Smart Ambulance guidance system by Bhushan Anant Ramani, Amrutha Jeyakumar [4] purposed a system in which is divided into three parts. First web application, the web application is designed for the ambulance driver. The application provides a user interface for the ambulance driver to choose a route and navigate the ambulance accordingly. The Ambulance location data is tracked using GPS and sent to the server. When the ambulanceis about to reach near the traffic signal, the ambulance driver can send the request to turn on the required lane to green. The data is sent to a server from which the traffic signal switches the lane to green for the ambulance to pass by. Second cloud server the cloud server is used which acts as the medium of communication between the traffic signal and the ambulance. Third traffic signal, Arduino Uno board is interfaced with Esp 8266 Wi-Fimodule which is used as a traffic signal. The Arduino module is programmed to fetch the data from the server. The traffic signal waits for the server if the server sends a request thenthe signal will turn green till ambulance crosses that signal.

Ambulance clearance Traffic Control System by R. Arulmozhi, A.G. Deviga, N. Ezhilarashi, P. jayanthirani [17] purposed a system which is implemented in three parts. First part contains automatic traffic control. Here, each vehicle is equipped with an RFID tag. When the ambulance comes in the range of RFID reader, it will send the signal to the RFID reader. The RFID reader will set the green light period for that track. Second part is for the emergency vehicle clearance. Here, each vehicle contains ZigBee transmitter module besides the ZigBee receiver which will remain implemented by the traffic junction. When the emergency vehicle comes in range of receiver it will send the signal through the ZigBee transmitter to the ZigBee receiver. It will make the traffic light to change to green. Once the ambulance passes through, the receiver no longer receives the ZigBee signal andthe traffic light is turned to red. The third part is responsible for stolen vehicle detection. Here, when the RFID reader reads the RFID tag, it compares it to the list of stolen RFIDs. If a match is found, it will send SMS to the police control room and changes the traffic light to red, so that the vehicle is made to stop in the traffic junction and police can take appropriate action.

A Novel Emergency Telemedicine System by Sotiris Pavlopouios, Alexander Berier [1] purposed a system in which the authors have developed a portable medical device, that allows telediagnosis, long distance support, and teleconsultation of mobile health care providers by experts located at an emergency coordination center or a specialized hospital. The system comprises of two separate modules, the mobile unit (ambulance site), where expert assistance is needed, and the consultation unit (hospital site) where experts are located and telemedicine directions are given. The control of the mobile station is fully automated. The system communications are performed via cellular networks using the GSM standard.

A jade based healthcare system by Sharmila Rathod [6], purposed a Jade-based Multidatabase decision making healthcare system , In this system if a person sees a catastrophic condition of a patient he immediately calls to a transmit center, with the information's about the accident's location, area. The most appropriate ambulatory service, gets a message from the transmit centre for dispatching an ambulance on the accident sight. The paramedics present in the ambulance makes use of PDA or a laptop in the ambulance to find out the list of near proximity hospitals from the accident position. Paramedic uses a 
details about the patients like patients aadhar ID, name, gender, type of treatment needed for that emergency position, which he acquire from a dispatch center. After doing this, the person in ambulance side utilizes the travelling time to reach the accident location by launching two mobile agents namely hospital mobile agent and a repository mobile agent. As per the characteristic of mobile agents, these two agents also possess feature such as autonomous and asynchronous, with which they gather the important information for a paramedic. Lastly all their result are collaborated and displayed on a PDA on ambulance. The hospital mobile agent salvages the most recent information about all the hospital recourses, among the listed hospital given to it. The Short listing a set of hospitals is done by a paramedic derived from the patient's location. If it is not specified then agent might visit to all the associated hospital in an application, regardless of its physical location from an accident scene.

Traffic Control Using Zigbee Module by Aadish Agarwal, Shreyash Saurabh [12] purposed a system in which two methods are implemented in this system. First is that as soon the emergency vehicle driver switches on the zigbee module transmitter and as soon as it startscommunicating with the zigbee module that will be installed in the other end at the traffic pole, the traffic light will turn green and as soon as the vehicle passes the junction, traffic signal will automatically convert to previous state as well as the timing in the traffic signal will be bring back to previous state. Second method is that every vehicle will be provided RFID tag while registration. In this information for that particular vehicle will be provided like unique vehicle identification as well as a tag which will help RFID reader to identify it is as emergency vehicle or a normal vehicle by providing $\mathrm{E}$ or $\mathrm{N}$ tag where $\mathrm{E}$ stands for emergency vehicle and $\mathrm{N}$ for normal vehicle. As $\mathrm{E}$ tag vehicle pass through traffic signal RFID reader that will be installed in traffic signal pole will identify and that lane will be made green. To find a stolen vehicle, the user has to contact the Transport office to update the database of the vehicle with $\mathrm{T}$ for Theft such that as soon as vehicle passes through any of the traffic signal junction RFID reader will identify the stolen vehicle by sending details to the traffic control centre via zigbee module and crosscheck the details with the Transport control unit database and on confirmation of the vehicle system control unit send alert to the nearby police station so that police can take immediate action.
Automatic Smart Ambulance System with Intelligent Traffic Control by N. Lakshmipriya,

S. Dineshkumar, M. Malathi, R. Pmaranisuga, A. Mohanraj [14] purposed a system in which the system contains IR transmitter and IR receiver which are mounted on the either sides of roads. The IR system gets activated whenever any vehicle passes on road between IR transmitter and IR receiver. The Microcontroller controls the IR system and counts number of vehicles passing on road. Microcontroller also store vehicles count in its memory. The traffic light is situated at a certain distance from the IR system. The concept of IR wave is used to provide passage to emergency vehicles, the IR transmitter is installed on every emergency vehicles. If the vehicle comes in range of IR receiver, the IR receiver will send a signal to microcontroller and the respective lane's light goes green. When a vehicle meet with an accident immediately the information will be transferred to control room. So, the rescue team can immediately trace the location from where the message came, and the patient health parameters are sent to a ambulance via serial communication and this data will be sent to the hospital server.

Intelligent Traffic Control \& Smart Ambulance by Saurabh Bharade, Pradnya Botre, Sayali Nagane, Mihir shah [2] purposed a system which is divided into two modules. First module is an android application. Whenever ambulance reaches to the accident spot, first the ambulance driver will feed the patient's information in the android application which consists of patient's name, age, blood group, gender, and the patient's parameter like ECG, blood pressure, etc. This information will be sent to the hospital's so that the hospital staff can be prepared for the requirements needed to the patient. After that, depending on patient's situation driver will send emergency or nonemergency command via android application to the server. This module works on the principle of IoT with the help of REST APIs. Also, Ambulance's current location and current direction of movement is sent to the server. Second module is the hardware module of traffic signal. In hardware module they have used Arduino for traffic signal. It consists of Wi-Fi module. With the help of Wi-Fi module, it captures information from the server. Because of this Wi-Fi module, the android application is directly connected to the traffic signal.

Smart Prioritized Ambulance Service by S. Naveen, C. 
Chitra [15] purposed the traffic control system which is cleared by using ZigBee sensor and RF transmitter and receiver. It is used to find the arrival of the ambulance. ZigBee Module is used for traffic control in case of ambulances arriving at the signal with the patient in critical condition. The arrival of ambulance data will be sent to the PIC controller by using ZigBee sensor. These data are transferred to server unit by using ZigBee. The data consists of temperature and heart beat sensors to monitor the patient's condition. RF Transmitter is used to transmit the signals. The signals are transferred using the GSM and ZigBee Module. The receiver unit consist of microcontroller, ZigBee module, RF receiver. The RF receiver receives the signals from the RF transmitter and immediately controls the traffic signals with the help of microcontroller and ZigBee modules. The path in which the ambulance arrives is cleared and the remaining signals are turned OFF (red).

Smart Ambulance Rescue System with Patient Health Monitoring by Irin Sherly S, Maria Sobitham, Manisha Nandhini K [3] purposed a system in which the health parameters of the patient body temperature, ecg, bpm are fetched through sensors and the patients' healthdata is transmitted to the hospital. The data transmission takes place with the help of Arduino board. The patients GPS location is automatically sent to the cloud and the admin server. The path for ambulance is provided using RF transmitter and receiver. The RF transmitter is inside the ambulance and RF signal is mounted on traffic signal. When the ambulance reaches in the range of RF receiver the signal is turned green for ambulance to pass. After the ambulance passes the signal is restored back to its original form.

Smart Ambulance and Traffic Controlling System by Sudhakara H.M, Girish H.R, Vinay Kumar, Kumar Swamy N, Sachin Kumar [5] purposed system which is a RFID-based system, which manages and regulates the traffic signals at junctions when the emergency vehicle approaches, by allowing the straight forward passage out of the traffic congestions. The ambulance is tracked by using GPS. This location is send to the application. The application performs the algorithm with the help of this data and so the google map. It controls the signals on its path. The ambulance must be equipped with the GPS System. This GPS System will send the coordinates of the vehicle at every moment to application Server. Each vehicle must be logged in to the android application. This application keeps tracking the vehicle and tracks the route. The server accepts all the knowledge, based on this information the server finds this location of the vehicle and also the route selected to the destination Whenever the vehicle comes within the space of certain meters from the signal the server must send the required action so as that the vehicle doesn't must wait at the signal. A symbol is additionally sent to the destination hospital so as that the hospital authorities are able to handle the patient. Hospitals also assign the priority to the patient supported their situation.

Traffic Signal Control System for Ambulance by Madhuri S Dabade, Shivani S Shete [19] purposed system which is designed to control the traffic signals along the path of the ambulance, when an ambulance approaches an intersection the traffic light is switched to green and as soon as the ambulance passes the intersection the control is restored to the proper signal again.it is IoTbased design for the system, it can be virtually be controlled from anywhere. It Makes it universally controllable and increases the responsive effectiveness. It can be used in situations like traffic congestion, emergency management, VIP escort etc. Thus the system increases the possibility of saving a life. The driver of the ambulance selects the route for the hospital, this route information is send to server, the control center then turns all the signals green along this route for ambulance to pass, after ambulance passes through these signals they are restored to their original state.

Model of autonomous car by Sharmila S. Gaikwad, Richa Koli, Uzma Kazi, Samiksha Mhadeshwar[15]. In this system, Pi Camera is mounted on top of the car which takes inputimages in high frame/second to feed the environment data live. These images are then processed in grayscale format using image processing to reduce the dimensional matrix required for RGB images. Using Convolutional Neural Network for image classification, the convolution layer finds patterns in the images which classify the various architectures of the road. These images are classified into Left, Right, Forward and Reverse for the movement of the car. These images are then firstly used for training the system under various circumstances. The training is done to make the system capable of predicting or classifying the actions to be taken for driving. The system uses combination of various sensors to detect object and the speed of the objects present on the road. After the decisionis made, 
the output is given as input to the Arduino Microcontroller. The Arduino controls the DC brake system and directs it or controls its speed.

Intelligent Traffic Control System for Ambulance by Apurva Bondade, Nikita Wasnik, Bhairavi Karale [10] purposed a system which is implemented in three modules. The first module is fully responsible for providing the route to the drivers of the ambulance. It will also monitor and track the ambulance from where it is arriving and where it is going. The second module is responsible for sending the request to the admin to get permission to reach to the location. As soon as the driver sends request he will have to wait for the route. After the driver selects the route, the driver will then send request to the cloud database to create green corridor.in third module it is dedicated for the manipulation of the signals. As soon as the cloud gets the request, the cameras will be activated for image processing. Once the ambulance is spotted then it will verify and provide a green corridor. The driver will receive the notification and will select path of the source and the destination.

\section{CRITICAL ANALYSIS AND REVIEW}

After reading many papers thoroughly we understood that the main parts of emergency medical services are transmitter, receiver and microcontroller. Indeed, these systems help in getting the medical help to the patient but many systems will waste a lot of time reaching hospital due to traffic or if there is no internet available in the region. Also, we think that the proposed systems by some authors can be more beneficial if the patient database contains patient's medical history such as blood group, heart rate, previous medical records, etc so that the medical professionals can be well equipped beforehand and can treat the patients faster. Also, if the system warns the driver of bad weather, and conditions of roadsahead then driver can avoid that route and take another faster route for the hospital.

\section{CONCLUSION}

Thus, in this paper we have discussed the different methodologies that are used in providing green corridor for the ambulances in traffic. The $\mathrm{Rf}$ based smart traffic control system provides an answer to the traffic congestion problem and this can be also an efficient method to provide path for the emergency vehicles, and taking vital health parameters and sharing it with nearby hospital.

\section{ACKNOWLEDGEMENT}

We wish to express our sincere gratitude to Dr. Sanjay U. Bokade, Principal and Prof. SunilP Khachne, H.O.D of Department Computer Engineering of Rajiv Gandhi Institute of Technology for providing us an opportunity to do our project work. This project bears on imprint of many people. We sincerely thank our project guide Dr. Sharmila Gaikwad for her guidance and encouragement in carrying out this synopsis work. Finally, we would like to thank our colleagues and friends who helped us in completing project work successfully

\section{REFERENCES}

[1] S. Pavlopoulos, S. Dembeyiotis, G. Konnis, D. Koutsouris, "AMBULANCE - Mobile Unit for Health Care Provision via Telematics Support," Proceedings of the IEEE Engineering in Medicine \& Biology, Amsterdam, The Netherlands, October 1996.

[2] Linganagouda R, PyintiRaju, Anusuya Patil"Automatic Intelligent Traffic Control System" IJAREEIE, Vol.5, Issue7, July2016.

[3] Hooman Samani, Rongbo Zhu,"Robotic Automatetd External Defibrillator Ambulance for Emergency Medical Service in Smart Cities", IEEE Access, Volume: 4, 2016.

[4] RajeshwariSundar, SanthoshsHebbar and VaraprasadGolla 'Implementing intelligent traffic control system for congestion control,ambulance clearance, and stolen vehicle detection'-IEEE sensors journal, Vol. 15,no. 2016.

[5] Siva Kumar Avula, Cheng Siong Lim, Shubhangi C Deshmukh, IEEE "Impact of Ambulance Dispatch Policies on Performance of Emergency Medical Services", December2011.

[6] Sharmila Gaikwad, "A Jade Based Healthcare system”, IJCS Journals, Volume 3, Issue 1, No 1, 2015 Reference ID: IJCS-071, PP 407-412.

[7] Ruihua Zhang, and Dongfeng Yuan, "A Health Monitoring System for Wireless Sensor Networks," in Proc. of 2ed IEEE Conference on Industrial Electronics and Applications (ICIEA), pp. 1648-1652, Harbin, China, May 2007. 
[8] Akshay Vishawanath, Akshay Vishwanath, Lalit Bhosle, Rishab Bhandari, Sharmila Gaikwad, "Internet Controlled Vehicle", International Journal of Recent and Innovation Trends in Computing and Communication, Published in IJRITCC Journal, Volume 4, Issue 4, April 2016, ISSN: 2321-8169 Scientific Journal Impact Factor: 5.837

[9] Veeramuthuvenkatesh, M.prashanthkumar, V.Vaithayanathan, Pethuru Raj," An ambient healthmonitor for the new generation healthcare," Journal of Theoretical and Applied Information Technology, Vol. 31 No.2, pp. 9199,Sep 2011.

[10] Buchenscheit, Andreas, et al. "A VANET-based emergency vehicle warning system." Vehicular Networking Conference (VNC), 2009 IEEE, 2009.

[11]A. Attar, P. Chaudhary, P. Dhuri, K. Vaidya, and N. Venkatesan, "Intelligent traffic management system," International Education and Research Journal, vol. 1, no. 4, pp. 1-3,2015.

[12] Saurabh Kapoor, Parul Gupta, Pooja Sharma, Prabhu Nath Singh, "Intelligent Ambulance with Automatic Traffic Control", International Research Journal of Engineering and Technology (IRJET), Apr -2017.

[13] Sharmila Gaikwad, "Mobile Agents in Heterogeneous Database Environment for Emergency Healthcare System", at ITNG 2008 5th International Conference on Information Technology- New Generations April 7-9, 2008, Las Vegas, Nevada, USA. Published in IEEE Computer society digital library, ISBN:978-07695-3099-4, PP.1220- 1221

[14] Linganagouda R, PyintiRaju, Anusuya Patil"Automatic Intelligent Traffic Control System" IJAREEIE, Vol.5, Issue7, July2016.

[15] Sharmila Gaikwad, Richa Koli, Uzma Kazi , Samiksha Mahadeshwar,"Model of Autonomous Car". International Journal of Engineering Research \& Technology (IJERT)

ISSN: 2278-018 IJERT,Vol. 9 Issue 05, May-2020

[16] Vahedha and Dr.B.NagaJyothi (2016) 'Smart traffic control system for clearance to emergency vehicles using arduino software'- International Journal of Technical Research and Applications, Volume 4, Issue 3 PP. 307-309

[17]C. Kotronis et al., "Managing Criticalities of eHealth IoT systems," IEEE Wireless Broadband (ICUWB), Salamanca, 2017.
[18]S. Hebbar, P. Pattar, and V. Golla, "A mobile zigbee module in a traffic control system," Potentials, IEEE, vol. 35, no. 1, pp. 19-23, 2016

[19]Malik Tubaishat, Yi Shang and Hongchi Shi -Adaptive Traffic Light Control with Wireless Sensor Networksll Consumer communication and network conference, 2007CCNC 4th IEEE. 